\title{
The curious tale of perioperative precision medicine: a story of hydroxocobalamin and cardiac surgery-associated vasoplegia
}

\author{
Miklos D. Kertai, MD, PhD • Andrew D. Shaw, MB, FRCA, FFICM, FCCM
}

Received: 2 January 2018/Accepted: 2 January 2018/Published online: 8 February 2018

(c) Canadian Anesthesiologists' Society 2018

Currently, most prevention strategies and therapies are designed for the average patient, and patients are generally not matched with the prevention or treatment strategies that will work best for them. The problem with this approach is that patients are not clones of each other and do not respond in the same way to any given treatment. Clinical trials generally report average treatment effects and are not sensitive to heterogeneity whereby a treatment may be beneficial in one subgroup but ineffective, or even harmful, in another.

In recognition of this problem, advocates of an approach for disease prevention and treatment that takes into account individual variability in patient characteristics (e.g., genetic, environment, lifestyle) are promoting the emerging concept of personalized or precision medicine. ${ }^{1}$ In several areas of medicine, examples of the role of precision medicine in day-to-day clinical care are emerging. These include targeted approaches to treating the underlying causes of specific genetic variants in cystic fibrosis, ${ }^{2}$ pharmacogenomic-guided methods of warfarin dosing, ${ }^{3}$ prediction of response to clopidogrel, ${ }^{4}$ and molecular testing of cancer patients for selection of a more targeted chemotherapy approach. ${ }^{5}$

\footnotetext{
M. D. Kertai, MD, PhD

Division of Cardiothoracic Anesthesiology, Department of Anesthesiology, Vanderbilt University Medical Center, 1211, 21st Avenue South, Medical Arts Building, Room 722,

Nashville, TN 37212, USA

\author{
A. D. Shaw, MB, FRCA, FFICM, FCCM ( $\square)$ \\ Department of Anesthesiology and Pain Medicine, 2-150M \\ Clinical Sciences Building, University of Alberta, Edmonton, \\ AB T6G 2G3, Canada \\ e-mail: ashaw2@ualberta.ca
}

In contrast, the role of precision medicine in the perioperative setting (perhaps for the prevention or treatment of perioperative adverse events) has not been as widely explored. ${ }^{6}$ Indeed, the overarching premise of precision perioperative medicine would be to identify and stratify individual patients into categories based on patients' susceptibility to develop certain adverse perioperative events. The differing pathophysiology and prognosis of these adverse perioperative events or the individual responses of patients to specific preventative treatment and/or intervention strategies might then be considered throughout the perioperative continuum. Subsequently, these strategies could be combined with genetic and molecular approaches and/or with information from measuring biomarkers of end-organ injury for further risk stratification and refinement. Then, an individual patient risk profile might be established and continuously updated leading to a more targeted preventative or therapeutic decision-making approach in those patients at increased risk for adverse perioperative events. Theoretically, this could lead to reduced healthcare expenses and avoidance of treatment-related side effects and/or complications in patients at lower risk for adverse perioperative outcomes or in whom selected treatments are known to be ineffective.

In this issue of the Journal, Shah et al. ${ }^{7}$ report their experience with hydroxocobalamin $\left(\mathrm{B}_{12}\right)$ infusion as a potential rescue agent for refractory vasoplegia during or immediately following cardiopulmonary bypass (CPB). They studied 33 patients who underwent cardiac surgery with the aim of identifying a group of patients in whom this treatment could provide some benefit.

The authors identified cardiac surgical patients $18 \mathrm{yr}$ and older who had an emergency pharmacy order for intravenous methylene blue (MB) infusion and/or $B_{12}$ 
infusion. As per the authors' institutional protocol, infusion of $\mathrm{MB}$ and/or vitamin $\mathrm{B}_{12}$ was indicated and initiated when the attending surgeon and anesthesiologist agreed that there was significant potential risk from ongoing hemodynamic instability leading to vasodilatory shock. This rescue therapy was initiated if the patient was unable to sustain a mean arterial pressure (MAP) $>65 \mathrm{mmHg}$ despite 0.1 $\mu \mathrm{g} \cdot \mathrm{kg}^{-1} \cdot \mathrm{min}^{-1} \quad$ norepinephrine, $0.04 \quad$ unit $\cdot \mathrm{min}^{-1}$ vasopressin, and $0.1 \mu \mathrm{g} \cdot \mathrm{kg}^{-1} \cdot \mathrm{min}^{-1}$ epinephrine support. Subsequently, these patients received $\mathrm{MB}$ and/or $\mathrm{B}_{12}$ infusion administered as an intravenous bolus over 15 min during or immediately following CPB.

The authors used semi-parametric group-based mixture models to identify patient clusters of MAP responses following $\mathrm{B}_{12}$ administration. These models were fitted to MAP measurements sampled at five-minute intervals from the time of $\mathrm{B}_{12}$ infusion until $110 \mathrm{~min}$ post-infusion. Of the 33 patients, $20(61 \%)$ received $B_{12}$ during $C P B$ and 13 (39\%) received it immediately following CPB. Intravenous MB infusion was administered to 15 patients (45\%), two patients $(13 \%)$ received it before the start of $\mathrm{CPB}$, ten patients $(67 \%)$ received it during $\mathrm{CPB}$, and three patients (20\%) received it immediately following CPB. There was also a group of 12 patients who received $\mathrm{MB}$ prior to $\mathrm{B}_{12}$ intravenous administration and three patients received it after $\mathrm{B}_{12}$ intravenous administration.

The authors were able to identify and separate patients into four groups based on their MAP response: "poor responders", "responders", "sustainers", and "rebounders". As characterized by their respective MAP response, the "poor responders" showed little or no immediate response to $\mathrm{B}_{12}$ intravenous administration, whereas the "responders" patient group showed a brisk response in MAP to $\mathrm{B}_{12}$ intravenous administration followed by sustained maintenance of MAP. The third "sustainers" group of patients were described as showing a small constant increase in MAP following $\mathrm{B}_{12}$ intravenous administration. The fourth group of "rebounders" were identified by an apparent hypertensive overshoot immediately after $B_{12}$ intravenous administration followed by a decline in MAP to $65 \mathrm{mmHg}$ over the next hour. As one might expect, compared with patients in the other groups, patients in the poor responder group were at higher risk for mortality. They also had relatively longer surgery and CPB times, durations of mechanical ventilation, and length of intensive care unit stay, as well as showing little change in vasopressor use following $\mathrm{B}_{12}$ intravenous administration. In contrast, patients in the responder group required relatively shorter ventilation time and length of intensive care unit stay, despite being at an elevated risk for mortality; they also showed a significant reduction in vasopressor use after $\mathrm{B}_{12}$ intravenous administration. Patients in the sustainer and rebounder groups were at lower risk for mortality and required relatively shorter ventilation time and length of intensive care unit stay, and they had either little or no reduction in vasopressor use (sustainers) or a significant reduction in vasopressor use (rebounders).

The case series presented by Shah et al. ${ }^{7}$ illustrates how a combination of patient- and procedure-related characteristics might identify patients at risk for developing cardiac surgery-associated vasoplegia and how such patients could be grouped according to their MAP responses when treated with $B_{12}$ therapy. Nevertheless, given that this was a retrospective case series, the authors could not explore whether the four different groups of phenotypes in MAP responses were explained by some genetically driven individual characteristics or molecular signatures of the patients. Further, as also acknowledged by the authors, this case series had additional important limitations that would influence the immediate translation of their findings to the management of patients with cardiac surgery-associated refractory vasoplegia, and this will have to be addressed in future studies. As this study did not include a group of patients as controls, the authors were unable to differentiate whether refractory hypotension was due to low vascular resistance, low cardiac output, or a central-peripheral blood pressure gradient. There were also differences in sequence and timing of intravenous $\mathrm{MB}$ and $\mathrm{B}_{12}$ intravenous administration in individual patients, and for consistency vasopressor doses were expressed in norepinephrineequivalent units. Nevertheless, this method largely ignores differences in the pharmacodynamics and modes of action of the different vasopressor agents or their susceptibility to pharmacogenomic variability. 8,9 Moreover, charting of vasopressor use was not uniform across data logging systems, and bolus dosages were frequently omitted from case records leading to a potential underestimation of the total vasopressor doses.

In summary, these initial data reported by Shah et al. highlight several important lessons on how future studies attempting to link specific patient-related characteristics or treatment modalities with perioperative and long-term outcomes and/or adverse drug responses might be conceived and conducted. Future studies should be designed using a comprehensive framework that includes ${ }^{10}$ :

1) a standardized definition(s) of the phenotypes of interest (including short- and long-term adverse outcomes such as physiologic perturbations, organ injury or dysfunction, adverse drug responses, transition from acute to chronic pain). This may be facilitated by developing and refining electronic medical record-driven phenotyping algorithms; 
2) careful characterization of the underlying genetic variants and identification of the mechanisms linking a specific genotype to phenotype;

3) development and validation of actionable diagnostics implemented at the point of care using electronic medical record-integrated best practice and clinical decision support tools.

Subsequently, knowledge gained through the conduct of such studies could be translated to prospective risk profiling that incorporates information on genetic predictors of adverse drug responses and/or postoperative outcomes. This might have potential implications for patient-specific additional preoperative testing. This then could be followed by physiologic optimization and interventions, personalized choices of drugs and dosage regimens, choice of perioperative monitoring strategies, type and extent of surgical intervention, and postoperative care resource utilization. Eventually these future studies will fulfill the premise of perioperative precision medicine by providing perioperative physicians with far more robust information to use in designing the most appropriate and safest perioperative risk assessment and management for an individual patient.

\section{L'étrange conte de la médecine de précision périopératoire : une histoire de l'hydroxocobalamine et de la vasoplégie associée à la chirurgie cardiaque}

Actuellement, la majorité des stratégies préventives et des traitements sont conçus pour le «patient moyen » et d'une manière générale, les patients ne seront pas appariés aux stratégies de prévention ou de traitement qui vont être les meilleures pour eux. Cette démarche soulève un problème : les patients ne sont pas des clones les uns des autres et ne répondent pas de la même façon à un traitement donné. Les essais cliniques décrivent habituellement les effets d'un traitement moyen et ils ne sont pas sensibles à une hétérogénéité qui fait qu'un traitement peut être bénéfique pour un sous-groupe, mais inefficace, voire nocif, pour un autre.

Reconnaissant ce problème, des défenseurs d'une démarche de prévention et de traitement des maladies tenant compte de la variabilité individuelle des caractéristiques des patients (par exemple, la génétique, l'environnement, le mode de vie) militent pour un nouveau concept de médecine personnalisée ou de médecine de précision. ${ }^{1}$ Des exemples de la place de la médecine de précision commencent à apparaître dans les soins cliniques quotidiens dans plusieurs champs de la médecine. Ce sont notamment des approches ciblées du traitement des causes sous-jacentes de différentes variantes génétiques spécifiques de la fibrose kystique, ${ }^{2}$ des méthodes de dosage de la warfarine guidées par la pharmacogénomique, ${ }^{3}$ de prédiction de la réponse au clopidogrel, ${ }^{4}$ et des tests moléculaires pour la sélection d'une chimiothérapie ciblée chez des patients cancéreux. ${ }^{5}$

En revanche, la place de la médecine de précision dans un contexte périopératoire (pour la prévention ou le traitement d'événements indésirables périopératoires, par exemple) n'a pas été largement explorée. ${ }^{6}$ En fait, une prémisse fondamentale de la médecine de précision périopératoire serait d'identifier et de catégoriser les patients individuels en fonction de leur susceptibilité de développer certains événements indésirables périopératoires. $\mathrm{La}$ multiplicité des processus physiopathologiques et des pronostics de ces différents événements périopératoires ou des réponses de chaque patient aux stratégies spécifiques de prévention et/ou d'intervention pourrait alors être envisagée tout au long du continuum périopératoire. Dans un deuxième temps, ces stratégies pourraient être combinées à des démarches génétiques et moléculaires et/ou à l'information provenant du dosage de biomarqueurs de lésions des organes cibles pour une stratification et un affinement encore plus poussés du risque. Il serait alors possible d'établir un profil de risque de chaque patient et de le mettre continuellement à jour, aboutissant à des démarches de prise de décisions en matière de prévention et de traitement encore plus ciblées pour les patients à risque élevé d'événements indésirables périopératoires. Théoriquement, cela pourrait déboucher sur une baisse des dépenses de santé et sur l'évitement d'effets indésirables et/ou de complications liées au traitement chez les patients à plus faible risque d'événements périopératoires ou chez lesquels on sait que les traitements sélectionnés sont inefficaces.

Dans ce numéro du Journal, Shah et coll. ${ }^{7}$ rapportent leur expérience de la perfusion d'hydroxocobalamine $\left(\mathrm{B}_{12}\right)$ comme agent de secours éventuel pour la vasoplégie réfractaire pendant ou immédiatement après une circulation extracorporelle (CEC). Ils ont étudié 33 patients ayant bénéficié d'une chirurgie cardiaque dans le but d'identifier un groupe de patients pour lesquels ce traitement pourrait constituer un certain avantage.

Les auteurs ont identifié des patients de chirurgie cardiaque âgés d'au moins 18 ans pour lesquels il y avait eu une demande pharmaceutique urgente pour du bleu de méthylène $(\mathrm{BM})$ et/ou de $\mathrm{B}_{12}$ en perfusion. Selon le 
protocole de l'établissement des auteurs, une perfusion de $\mathrm{BM}$ et/ou de vitamine $\mathrm{B}_{12}$ était indiquée et commencée quand le chirurgien pratiquant l'intervention et l'anesthésiologiste étaient d'accord sur l'existence d'un risque significatif de choc par vasodilatation due à l'instabilité hémodynamique constatée. Ce traitement de secours était instauré si le patient ne parvenait pas à maintenir une pression artérielle moyenne (PAM) $>65 \mathrm{mmHg}$ malgré un soutient par $0,1 \mu \mathrm{g} \cdot \mathrm{kg}^{-1} \cdot \mathrm{min}^{-1}$ de norépinéphrine, 0,04 unité $\min ^{-1}$ de vasopressine et $0,1 \mu \mathrm{g} \cdot \mathrm{kg}^{-1} \cdot \mathrm{min}^{-1}$ d'adrénaline. En conséquence, ces patients recevaient une perfusion de $\mathrm{BM}$ et/ou de $\mathrm{B}_{12}$ administrée sous forme de bolus en $15 \mathrm{~min}$ pendant ou immédiatement après la CEC.

Les auteurs ont utilisé un mélange de modèles reposant sur des groupes semi-paramétriques pour identifier les groupes de patients selon la réponse de la PAM après l'administration de $\mathrm{B}_{12}$. Ces modèles ont été adaptés à la prise de mesures de la PAM à intervalles de 5 minutes entre le moment de la perfusion de $\mathrm{B}_{12}$ jusqu'à 110 minutes après la perfusion. Sur les 33 patients, 20 (61\%) ont reçu la $\mathrm{B}_{12}$ au cours de la CEC et 13 (39\%) l'ont reçu immédiatement après la CEC. Une perfusion IV de BM a été administrée à 15 patients (45\%): deux patients $(13 \%)$ l'ont reçue avant le début de la CEC, dix patients $(67 \%)$ l'ont reçu pendant la CEC et trois patients (20 \%) l'ont reçue immédiatement après la CEC. Un autre groupe de 12 patients a reçu le BM avant l'administration intraveineuse de $B_{12}$ et trois patients l'ont reçu après l'administration intraveineuse de $\mathrm{B}_{12}$.

Les auteurs ont été capables d'identifier et de répartir les patients en quatre groupes en fonction de la réponse de leur PAM : «mauvaise réponse », « réponse », « maintien durable » et «rebond ». Comme l'indiquaient les réponses de leurs PAM, les patients du groupe « mauvaise réponse » ne présentaient pas ou peu de réponse immédiate à l'administration intraveineuse de $\mathrm{B}_{12}$ alors que la PAM des patients du groupe « réponse » répondait brusquement à cette administration, puis la PAM se maintenait durablement. Le troisième groupe de patients à « maintien durable » était décrit comme présentant une petite augmentation constante de la PAM après l'administration intraveineuse de $B_{12}$. Le quatrième groupe de patients à « rebond» a été identifié par un rebond hypertensif apparent immédiat après l'administration intraveineuse de $\mathrm{B}_{12}$ suivi d'un déclin de la PAM à $65 \mathrm{mmHg}$ au cours de l'heure qui suivait. Comme on pouvait s'y attendre, les patients affichant une mauvaise réponse avaient un plus grand risque de mortalité que ceux des autres groupes. La durée de l'intervention chirurgicale était également relativement plus longue chez ces patients, de même que les durées de CEC, de ventilation mécanique et de séjour en unité de soins intensifs; l'utilisation d'agents vasopresseurs était également peu modifiée après l'administration i.v. de $\mathrm{B}_{12}$. En revanche, les patients du groupe «répondeur» nécessitaient une durée de ventilation relativement plus courte, avec une durée de séjour en USI réduite, bien que présentant un risque élevé de mortalité; ils présentaient également une réduction significative de la consommation d'agents vasopresseurs après l'administration intraveineuse de $\mathrm{B}_{12}$. Les patients des groupes «maintien durable » et « rebond» avaient un plus faible risque de mortalité et nécessitaient des durées de ventilation et de séjour en unité de soins intensifs plus courtes; par ailleurs, leur recours aux agents vasopresseurs était peu ou pas réduit (groupe « maintien durable ») ou significativement réduit (groupe « rebond »).

La série de cas présentée par Shah et coll. ${ }^{7}$ illustre comment une combinaison de caractéristiques liées au patient et à la procédure pourrait identifier des patients à risque de développer une vasoplégie associée à la chirurgie cardiaque et comment ces patients peuvent être groupés en fonction de la réponse de leur PAM quand ils recevaient un traitement par $\mathrm{B}_{12}$. Néanmoins, considérant qu'il s'agissait d'une série de cas rétrospectifs, les auteurs n'ont pas pu explorer si la réponse de la PAM dans les quatre différents groupes était expliquée par des caractéristiques génétiques individuelles ou si ces patients présentaient des signatures moléculaires. De plus, comme le reconnaissent également les auteurs, cette série de cas présentait d'autres limitations importantes susceptibles d'influencer la transposition immédiate de leurs constatations dans la gestion de la vasoplégie réfractaire associée à la chirurgie cardiaque et qui devront être abordées dans des études futures. Cette étude ne comportant pas de groupe témoin de patients, les auteurs n'ont pas été en mesure d'établir si l'hypotension réfractaire était due à une faible résistance vasculaire, à un débit cardiaque bas ou à un gradient de pression sanguine centrale/périphérique. Il y a eu également des différences dans la succession et le moment opportun des administrations intraveineuses de $B M$ et de $B_{12}$ aux différents patients et les doses des agents vasopresseurs ont été exprimées en unités de norépinéphrine-équivalent, dans un but d'harmonisation. Mais cette méthode ne tient quasiment aucun compte des différences pharmacodynamiques et des modes d'action des différents agents vasopresseurs ou de leur susceptibilité à la variabilité pharmacogénomique. ${ }^{8,9}$ De plus, l'enregistrement de l'utilisation des agents vasopresseurs n'a pas été uniforme selon les systèmes d'entrée de données et les concentrations des bolus ont été souvent omises dans les dossiers, débouchant sur une possible sous-estimation des doses totales d'agents vasopresseurs..

En résumé, ces données initiales publiées par Shah et coll. soulignent plusieurs leçons importantes à retenir pour 
la conception et la réalisation d'études futures qui tenteront d'associer des caractéristiques spécifiques liées aux patients ou des modalités de traitement avec les aboutissements périopératoires et à long terme et/ou les réponses indésirables aux médicaments. Les études futures devront être conçues selon un cadre complet incluant ${ }^{10}$ :

1) Une ou des définitions standardisées des phénotypes d'intérêt (y compris les critères d'évaluation des effets indésirables à court et long terme, tels que les perturbations physiologiques, les lésions ou dysfonctionnements d'organes, les réponses indésirables aux médicaments, la transition de la douleur aiguë à la douleur chronique). Cela peut être facilité en élaborant et raffinant les algorithmes de phénotypage s'appuyant sur les dossiers médicaux électroniques;

2) Une identification précise des variantes génétiques sous-jacentes et l'identification des mécanismes liant un génotype spécifique à un phénotype;

3) Le développement et la validation d'outils diagnostiques utilisables et mis en œuvre au point de service qui font appel aux meilleurs outils de soutien à la pratique et à la décision clinique intégrés aux dossiers médicaux électroniques.

Ultérieurement, les connaissances acquises grâce à la réalisation de ces études pourront être transposées dans un profilage prospectif des risques qui incorporera l'information sur les éléments prédictifs génétiques de réponses indésirables aux médicaments et/ou les aboutissements postopératoires. Cela pourrait avoir des implications sur d'éventuels tests supplémentaires préopératoires spécifiques, puis être suivi par une optimisation et des interventions physiologiques, des choix personnalisés de médicaments et de schémas posologiques, un choix des stratégies de surveillance périopératoire, du type et de l'étendue des interventions chirurgicales, et dans l'utilisation des ressources de soins postopératoires. Éventuellement, ces études futures s'acquitteront des prémisses de la médecine de précision périopératoire en fournissant aux médecins intervenant à ce stade beaucoup plus de renseignements solides à utiliser pour la conception de l'évaluation du risque la plus adaptée et la plus sécuritaire et pour la gestion de chaque patient individuel.
Conflicts of interest None declared.

Editorial responsibility This submission was handled by $\mathrm{Dr}$. Hilary P. Grocott, Editor-in-Chief, Canadian Journal of Anesthesia.

Conflits d'intérêts Aucun déclaré.

Responsabilité éditoriale Cet article a été traité par le $\mathrm{D}^{\mathrm{r}}$ Hilary $\mathrm{P}$. Grocott, Rédacteur en chef, Journal canadien d'anesthésie.

\section{References}

1. The Precision Medicine Initiative (PMI) Working Group. Report to the Advisory Committee to the Director, NIH. The Precision Medicine Medicine Initiative Cohort Program - Building a Research Foundation for $21^{\text {st }}$ Century Medicine, September 2015. Available from URL: https://www.nih.gov/sites/default/files/ research-training/initiatives/pmi/pmi-working-group-report-2015 0917-2.pdf (accessed January 2018).

2. Kim J, Davies Z, Dunn C, Wine JJ, Milla C. Ivacaftor restores CFTR-dependent sweat gland fluid secretion in cystic fibrosis subjects with S945L alleles. J Cyst Fibros 2017. DOI: https://doi. org/10.1016/j.jcf.2017.12.005.

3. Ramirez AH, Shi Y, Schildcrout JS, et al. Predicting warfarin dosage in European-Americans and African-Americans using DNA samples linked to an electronic health record. Pharmacogenomics 2012; 13: 407-18.

4. Delaney JT, Ramirez AH, Bowton E, et al. Predicting clopidogrel response using DNA samples linked to an electronic health record. Clin Pharmacol Ther 2012; 91: 257-63.

5. Sluga R, Van Den Born BE, Roepman P, Peters BJ, Kastelijn EA, Schramel $F M$. Utilization of molecular testing and survival outcomes of treatment with first- or second-line tyrosine kinase inhibitors in advanced non-small cell lung cancer in a Dutch population. Anticancer Res 2018; 38: 393-400.

6. Shaw A, VandeVen T. Secrets of the perioptome: new tools for a new concept. Curr Opin Crit Care 2010; 16: 365-70.

7. Shah PR, Reynolds PS, Pal N, Tang D, McCarthy H, Spiess BD. Hydroxocobalamin for the treatment of cardiac surgeryassociated vasoplegia: a case series. Can J Anesth 2017. DOI: https://doi.org/10.1007/s12630-017-1029-3.

8. Kohli U, Hahn MK, English BA, et al. Genetic variation in the presynaptic norepinephrine transporter is associated with blood pressure responses to exercise in healthy humans. Pharmacogenet Genomics 2011; 21: 171-8.

9. Ghimire $L V$, Kohli $U, L i C$, et al. Catecholamine pathway gene variation is associated with norepinephrine and epinephrine concentrations at rest and after exercise. Pharmacogenet Genomics 2012; 22: 254-60.

10. Podgoreanu $M V$, Kertai MD. Genomic and precision medicine in anesthesia practice. In: Longnecker DE, Newman MF, Zapol WM, Mackey SC, Sandberg WS, editors. Anesthesiology -. 3rd ed. NY: McGraw-Hill Education; 2018. p. 1422-38. 